\title{
Yearly Electricity Consumption Forecasting using a Nonhomogeneous Exponential Model Optimized by PSO Algorithm
}

\author{
Xiaomin Xu1 ${ }^{1}$, Dongxiao Niu ${ }^{1}$, Ming Meng ${ }^{2, *}$ and Huifeng Shi ${ }^{3}$ \\ ${ }^{1}$ School of Economics and Management, North China Electric Power University, 102206, Beijing, China \\ ${ }^{2}$ School of Economics and Management, North China Electric Power University, 071003, Baoding, Hebei, China \\ ${ }^{3}$ Department of Mathematics and Phsics, North China Electric Power University, 071003, Baoding, Hebei, China
}

Received: 18 May. 2013, Revised: 22 Sep. 2013, Accepted: 23 Sep. 2013

Published online: 1 May. 2014

\begin{abstract}
Yearly electricity consumption trends of most developing countries usually exhibit approximately exponential growth curves. An optimized nonhomogeneous exponential model (ONEM) is proposed as a method of forecasting electricity consumption by using trend extrapolation. The parameters of the nonhomogeneous exponential equation are obtained by using the inverse accumulated generating operation, discretizing the differential equation, minimizing the residual sum of squares (RSS), and accumulating the homogeneous exponential equation. Furthermore, to improve forecasting precision, particle swarm optimization (PSO) algorithm is used to optimize the equation parameters. To evaluate the forecasting performance for comparison, the said model and two other traditional methods are used to forecast the yearly electricity consumption of India. Empirical results show that this model is much better than traditional methods for each error analysis indicator.
\end{abstract}

Keywords: Electricity Consumption Forecasting, Nonhomogeneous Exponential Model, PSO Algorithm

\section{Introduction}

Electricity consumption is one of the important driving factors of economic growth. The causal relationship between electricity consumption and economic growth has been proved by extensive research $[1,2,3,4]$. Unlike other energy sources, electricity cannot be stored on a large scale. Hence, precise forecasting of electricity consumption is very important to balance supply and demand. On the basis of the difference of forecasting indicators, research on electricity consumption forecasting is usually divided into two categories: short-term $[5,6,7,8]$ and mid/long-term $[9,10,11]$. As a representative research area of the latter, yearly electricity consumption forecasting plays an important role in electricity price adjustment and system expansion planning. Two methods of yearly electricity consumption forecasting are currently used. Some researchers focus on the relationship between the yearly electricity consumption and its influencing factors. They usually develop an equation to quantitatively simulate this relationship and obtain forecasting results by inputting the values of the influencing factors of the forecasting period into the equation $[12,13,14]$. Most of these methods usually simulate historical data efficiently. When used for forecasting, however, these methods do not usually produce satisfactory results because the exact values of the influencing factors in the forecasting period are unknown. Other researchers concentrate on the change rule of the electricity consumption trend itself. They use the historical electricity consumption data to build trend simulation equations and obtain the forecasting results by trend extrapolation $[15,16,17]$. The forecasting model proposed in this paper belongs to the latter.

The economic development of most developed countries is at the post-industrialization stage, and the electricity needs of people's daily lives have been met. Therefore, their yearly electricity consumption changes very slowly and is easily forecasted. By contrast, the yearly electricity consumption of developing countries, which is investigated in detail in this study, usually increases rapidly. In fact, from 2001 to 2010, among the

\footnotetext{
*Corresponding author e-mail: ncepumm@126.com
} 
top 30 electricity consumers of the world are 15 developed countries and areas whose yearly growth rate is only $1.1 \%$, whereas that of the other 15 developing consumers is as high as $7.4 \%$. In fact, for most developing countries, their long-term electricity consumption trends usually present approximately exponential growth curves [18].

Neural networks and other artificial intelligent algorithms have been used for trend extrapolation of daily $[19,20]$ and monthly [21,22] electricity consumption. Limited by the algorithms themselves, these models usually need large sample data with relative clear laws for parameter training. However, compared with daily and monthly electricity consumption, yearly data are very limited and, more importantly, affected by the socioeconomic and political environment. The intercept, slope, and curvature of a country's exponential growth curve do not usually remain stable for a long time. As a result, these models do not usually obtain satisfactory forecasting results.

At present, the $\operatorname{GM}(1,1)$ algorithm $[23,24,25]$ is widely used in extrapolating the approximate exponential curve. However, the forecasting equation of $\operatorname{GM}(1,1)$ is essentially homogeneous, that is, $\operatorname{GM}(1,1)$ is suitable only to a time series with a steadily increasing rate, which is a special form of the approximately exponential curve. This characteristic obviously does not necessarily exist in the yearly electricity consumption curve. To improve representativeness, a nonhomogeneous exponential model is proposed to extrapolate the electricity consumption trends of developing countries. Furthermore, to improve the forecasting results, particle swarm optimization (PSO) algorithm is used to optimize the equation parameters.

\section{Optimized Nonhomogeneous Exponential Model (ONEM)}

\subsection{Algorithm and limitation of $G M(1,1)$}

A nonhomogeneous exponential equation can be written as

$$
x^{(0)}(k)=c e^{a k}+b
$$

whereas its inverse accumulated generating operation (IAGO) equation is

$$
\begin{aligned}
y^{(0)}(k) & =x^{(0)}(k+1)-x^{(0)}(k) \\
& =c\left(e^{a}-1\right) e^{a k}
\end{aligned}
$$

Obviously, Eq. (2) is a homogeneous exponential equation.

As introduced before, $\operatorname{GM}(1,1)$ can simulate the homogeneous exponential curves. Hence, the $\operatorname{GM}(1,1)$ can be used to simulate the IAGO results of a nonhomogeneous exponential time series.

As a parameter lies in the exponential term of the homogeneous exponential equation, parameter estimations cannot be obtained directly by inputting the observational data and minimizing the residual sum of squares (RSS). The grey theory uses the differential form of the exponential equation, written as follows to estimate the parameters [23]:

$$
\frac{d y^{(1)}(t)}{d t}+\lambda_{1} y^{(1)}(t)=\lambda_{2}
$$

where $y^{(1)}(k)=\sum_{i=1}^{k} y^{(0)}(i)$ is the accumulated generating operation (AGO) time series.

To discretize Eq. (3), its first term is processed as

$$
\begin{gathered}
\frac{d y^{(1)}}{d t}=\lim _{\Delta t \rightarrow 0} \frac{y^{(1)}(t+\Delta t)-y^{(1)}(t)}{\Delta t} \\
\approx y^{(1)}(k+1)-y^{(1)}(k) \\
=y^{(0)}(k+1)
\end{gathered}
$$

and the second term as

$$
\lambda_{1} y^{(1)}(t)=\frac{\lambda_{1}}{2}\left[y^{(1)}(k)+y^{(1)}(k+1)\right]
$$

As a result, Eq. (3) is finally written as

$$
y^{(0)}(k+1)+\frac{\lambda_{1}}{2}\left[y^{(1)}(k)+y^{(1)}(k+1)\right]=\lambda_{2}
$$

Given the $\mathrm{n}$ sets of historical data, inputting them into Eq. (6) and minimizing the RSS results in the following estimations of $\lambda_{1}$ and $\lambda_{2}$ :

$$
\begin{aligned}
\hat{\lambda} & =\left(\mathbf{B}^{\mathrm{T}} \mathbf{B}\right)^{-1} \mathrm{~B}^{\mathrm{T}} \mathbf{Y} \\
& =\left[\hat{\lambda}_{1}, \hat{\lambda}_{2}\right]^{\mathrm{T}}
\end{aligned}
$$

where

$$
\mathbf{B}=\left[\begin{array}{ll}
-\frac{1}{2}\left[y^{(1)}(1)+y^{(1)}(2)\right] & 1 \\
-\frac{1}{2}\left[y^{(1)}(2)+y^{(1)}(3)\right] & 1 \\
\vdots & \vdots \\
-\frac{1}{2}\left[y^{(1)}(n-1)+y^{(1)}(n)\right] & 1
\end{array}\right], \mathbf{Y}=\left[\begin{array}{c}
y^{(0)}(2) \\
y^{(0)}(3) \\
\vdots \\
y^{(0)}(n)
\end{array}\right]
$$

Accordingly, the forecasting equation of $y^{(0)}$ is

$$
\hat{y}^{(0)}(k+1)=\left(1-\mathrm{e}^{\hat{\lambda}_{1}}\right)\left(y^{(0)}(1)-\frac{\hat{\lambda}_{2}}{\hat{\lambda}_{1}}\right) \mathrm{e}^{-\hat{\lambda}_{1} k}
$$

As shown in the aforementioned modeling process, to discretize the first term of the differential equation, Eq. (4) uses $y^{(1)}(k+1)$ and $y^{(1)}(k)$. However, there is no reason to use the mean of $y^{(1)}(k+1)$ and $y^{(1)}(k)$ for Eq. (5). In fact, for the second term of Eq. (3), the only certain fact is $t \in[k, k+1]$. The use of the mean can simplify the modeling process, but affects the precision of the estimated parameters. 


\subsection{Nonhomogeneous exponential equation}

Let $\hat{\beta}_{1}=\left(1-\mathrm{e}^{\hat{\lambda}_{1}}\right)\left(y^{(0)}(1)-\frac{\hat{\lambda}_{2}}{\hat{\lambda}_{1}}\right)$ and $\hat{\beta}_{2}=-\hat{\lambda}_{1}$ in Eq.(8) is further written as

$$
\hat{y}^{(0)}(k+1)=\hat{\beta}_{1} \mathrm{e}^{\hat{\beta}_{2} k} .
$$

Considering the relationship between $y^{(0)}(k)$ and $x^{(0)}(k)$, the forecasting equation of $x^{(0)}$ is

$$
\begin{gathered}
\hat{x}^{(0)}(k+1) \approx \hat{y}^{(0)}(k)+\hat{y}^{(0)}(k-1)+\cdots \hat{y}^{(0)}(2)+x^{(0)}(2) \\
=\hat{\beta}_{1} e^{\hat{\beta}_{2}(k-1)}+\hat{\beta}_{1} e^{\hat{\beta}_{2}(k-2)}+\hat{\beta}_{1} e^{\hat{\beta}_{2}}+x^{(0)}(2) \\
=\frac{\hat{\beta}_{1} e^{\hat{\beta}_{2}(k-1)}\left[1-e^{\hat{\beta}_{2}(k-1)}\right]}{1-e^{-\hat{\beta}_{2}}}+x^{(0)}(2) \\
=\frac{\hat{\beta}_{1} e^{\hat{\beta}_{2}(k-1)}}{1-e^{-\hat{\beta}_{2}}}+x^{(0)}(2)
\end{gathered}
$$

\subsection{Application of PSO}

The PSO algorithm proposed by Kennedy and Eberhart [26] is an optimization technique inspired by the life of natural swarms, such as birds and fishes [27]. It uses the learning, information sharing, and position updating strategy of each solution. With the behavior of the technique analogous to bird flocking and the search space analogous to the flocking area of the bird, each bird may be treated as a particle in the search space that is equipped with communicating abilities. To search candidates of interest in the domain, random particles are generated. These generated particles are further flagged with a certain velocity, and a communication system among the particles is established to enable them to converse with each other. This system is accomplished with the help of topologies and their mathematical formulations [28]. The main process of PSO algorithm is as follows:

A. Initializing the particle swarm. Let $t=0$, to ensure the optimization effect, the position of one of the particles is assigned to $[0.5,0.5, \ldots, 0.5]$, and other positions are stochastically assigned to vectors between 0 and 1 . Accordingly, the matrix $\mathbf{B}$ is written as

$\mathbf{B}=\left[\begin{array}{lr}-\left[z_{i}^{t}(1) y^{(1)}(1)+\left(1-z_{i}^{t}(1)\right) y^{(1)}(2)\right] & 1 \\ -\left[z_{i}^{t}(2) y^{(2)}(1)+\left(1-z_{i}^{t}(2)\right) y^{(1)}(3)\right] & 1 \\ \vdots & \vdots \\ -\left[z_{i}^{t}(n-1) y^{(1)}(n-1)+\left(1-z_{i}^{t}(n-1)\right) y^{(1)}(n)\right] & 1\end{array}\right]$

B. Performance evaluation. Based on the position of each particle, performance $F\left(z_{i}^{t}\right)$ is evaluated. In this model, the mean absolute percentage error (MAPE) (in $\%$ ) of the simulation to the historical data is selected as the indicator to measure the performance of each particle.

$$
\text { MAPE }=\frac{1}{N} \sum_{i}\left[\left|\frac{\hat{x}^{(0)}(i)-x^{(0)}(i)}{x^{(0)}(i)}\right|\right] \cdot 100
$$

where $x^{(0)}(i)$ is the $i^{\text {th }}$ yearly electricity consumption value and $\hat{x}^{(0)}(i)$ is its forecasting result.

C. Performance comparison and single position adjustment. The performance of each particle is compared with its best performance. If $F\left(z_{i}^{t}\right)<$ best $_{i}$, then

$$
\left\{\begin{array}{l}
\text { pbest }_{i}=F\left(z_{i}^{t}\right) \\
z_{\text {pbest }_{i}}=z_{i}^{t}
\end{array}\right.
$$

D. Performance comparison and all position adjustment. The performance of each particle is compared with the best performance of all particles. If $F\left(z_{i}^{t}\right)<$ gbest, then

$$
\left\{\begin{array}{l}
g \text { best }=F\left(z_{i}^{t}\right) \\
z_{\text {gbest }}=z_{i}^{t}
\end{array}\right.
$$

The speed vector of particles is adjusted

$$
v_{i}^{t}=v_{i}^{t-1}+\rho_{1}\left(z_{p \text { best }_{i}}-z_{i}^{t}\right)+\rho_{2}\left(z_{\text {gbest }}-z_{i}^{t}\right)
$$

where $\rho_{1}$ and $\rho_{2}$ are stochastic values. Particles are moved to the new positions

$$
\left\{\begin{array}{l}
z_{i}^{t}=z_{i}^{t-1}+v_{i}^{t} \\
t=t+1
\end{array}\right.
$$

Then, turning to step B until the performance is satisfactory.

\subsection{Modeling process of forecasting}

The modeling process of yearly electricity consumption forecasting by using ONEM is as follows:

A. Selecting the historical data. The electricity consumption data of a developing country for several continuous years are selected and written as $x^{(0)}$.

B. Data preprocessing. The IAGO time series, written as $y^{(0)}$, is obtained by using Eq. (2). Furthermore, the AGO time series of $y^{(0)}$ is calculated and written as $y^{(1)}$.

C. Designing several particles. The initial position of each particle is a vector. As introduced before, the initial position of one of the particles is assigned to $[0.5,0.5, \ldots, 0.5]$. Other initial positions are stochastically assigned to vectors between 0 and 1 .

D. Optimizing the parameter estimations. The optimum parameter estimations of Eq. (10) are obtained by using the PSO algorithm.

E. Forecasting the electricity consumption. The forecasting results of future electricity consumption are obtained by using Eq. (10).

\section{Model test}

\subsection{Data selection}

India is a large South Asian developing country that has experienced a high increase in gross domestic product 




Fig. 1: India's electricity consumption curve

over the past several decades [29]. The current lag of the basic industry has caused India to suffer from the effects of energy shortage. As per the published data from the Central Electricity Authority, India experienced an energy deficit of $8.5 \%$ and a peak demand shortage of $9.8 \%$ for the financial year 2010 to 2011. Of all the energy sources, electricity is experiencing the most severe shortage and has resulted in a massive loss to the national economy [29]. Precisely forecasting India's yearly electricity consumption will have great significance in adjusting energy-related policies and in promoting economic growth. Hence, India's yearly electricity consumption is selected to evaluate the performance of the proposed forecasting model. Real data (RD) of India's yearly electricity consumption during between 1971 and 2010 [18] were selected to draw the consumption curve (Fig. 1). As shown in Fig. 1, the long-term trend of India's electricity consumption curve is similar to an exponential growth curve. As a result of unexpected changes of the socioeconomic environment, the said curve exhibits several irregular waves.

\subsection{Forecasting results and error analysis}

The ONEM proposed in this paper was used to forecast India's yearly electricity consumption from 2001 to 2010. For each forecasting point, electricity consumption data for 20 continuous years that immediately precede the forecasting point were selected to build the forecasting model. For example, when forecasting the 2001 electricity consumption, electricity consumption data from 1981 to 2000 were selected to estimate the parameters of Eq. (10). The forecasting result was obtained by assigning $k=20$ in Eq. (10). Electricity consumption data from 2002 to 2010 were also forecasted by using the same method. Furthermore, to compare the forecasting performance, the $\operatorname{GM}(1,1)$ and neural

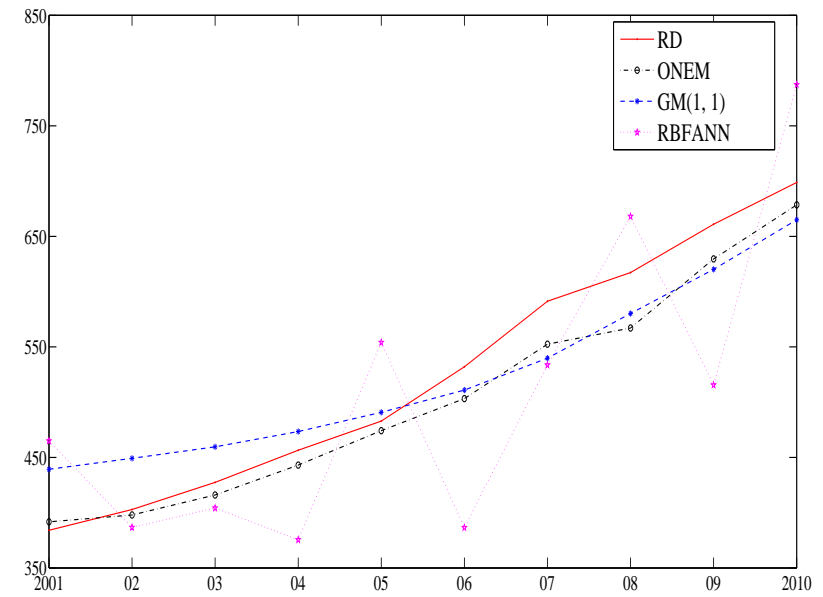

Fig. 2: Comparison among different forecasting results.

network model were used to forecast India's yearly electricity consumption from 2001 to 2010. For $\operatorname{GM}(1,1)$, the same as the ONEM, electricity consumption data for the 20 continuous years before the forecasting year were selected to build the model. For neural network models, the error backpropagation artificial neural network and radial basis function artificial neural network (RBFANN) are the most representative neural network models. As the latter is usually better than the former in terms of learning efficiency and stability [22], RBFANN was selected to forecast India's electricity consumption from 2001 to 2010. As with the ONEM and $\operatorname{GM}(1,1)$, electricity consumption data for 20 continuous years before the forecasting year were also selected to build the model. When training the RBFANN, for the 20 selected values, the 11 th to 20 th values were selected as the output values, respectively, and 10 values before each output value were selected as its input vector. After training, the forecasting result was obtained by inputting the 11th to 20th values into the network. The RD, forecasting results of each model, and their percentage errors (PE) are listed in Table 1.

To show the forecasting performance intuitively, the RD and its forecasting results were used to draw Fig.2

As shown in Fig.2, the irregular changes of the main trend have affected the generalization ability, which resulted in RBFANN having very unstable and obviously larger forecasting errors than the other two models in general. Compared with ONEM, the lack of a constant term results in a very smooth forecasting curve of $\mathrm{GM}(1$, 1). This characteristic renders GM unable to react quickly to irregular changes in the main trend.

To analyze the precision of the overall forecast, aside from the MAPE defined in Eq. (12), the median absolute percentage error (MdAPE) (in \%), maximum absolute percentage error (MaxAPE) (in \%), and geometric mean relative absolute error (GMARE) (in \%) were selected as indicators to compare the different forecasting models. 
Table 1: Real electricity consumption data and their forecasting results

\begin{tabular}{ccccccccccc}
\hline Year & 2001 & 2002 & 2003 & 2004 & 2005 & 2006 & 2007 & 2008 & 2009 & 2010 \\
\hline RD & 384.1 & 402.9 & 427.4 & 456.5 & 482.9 & 532.0 & 591.3 & 617.3 & 661.0 & 698.8 \\
ONEM & 391.7 & 397.9 & 416 & 443.1 & 474.2 & 503.2 & 552.5 & 567.1 & 629.6 & 678.5 \\
PE & 2 & -1.2 & -2.7 & -2.9 & -1.8 & -5.4 & -6.6 & -8.1 & -4.8 & -2.9 \\
GM $(1,1)$ & 439.3 & 449.2 & 459.6 & 473.4 & 490.8 & 510.9 & 539.8 & 580.2 & 620.2 & 665.0 \\
PE & 14.4 & 11.5 & 7.5 & 3.7 & 1.6 & -4.0 & -8.7 & -6.0 & -6.2 & -4.8 \\
RBFANN & 464.9 & 386.7 & 404.2 & 375.5 & 554 & 386.5 & 533.7 & 668.1 & 515.6 & 787.4 \\
PE & 21.0 & -4 & -5.4 & -17.7 & 14.7 & -27.3 & -9.7 & 8.2 & -22.0 & 12.7 \\
\hline
\end{tabular}

Table 2: Forecasting errors of each model

\begin{tabular}{ccccc}
\hline & MAPE & MdAPE & MaxAPE & GMARE \\
\hline ONEM & 3.84 & 2.92 & 8.13 & 23.31 \\
GM(1,1) & 6.84 & 6.09 & 14.37 & 41.74 \\
RBFANN & 14.29 & 13.70 & 27.35 & 87.13 \\
\hline
\end{tabular}

These indicators are defined as follows:

$$
\begin{gathered}
M A A P E=\underset{i}{\operatorname{madiam}}\left|\frac{\hat{x}^{(0)}(i)-x^{(0)}(i)}{x^{(0)}(i)}\right| \times 100 \\
\text { MaxAPE }=\max _{i}\left|\frac{\hat{x}^{(0)}(i)-x^{(0)}(i)}{x^{(0)}(i)}\right| \times 100 \\
\text { GMARE }=\left[\prod_{i}^{N}\left|\frac{\hat{x}^{(0)}(i)-x^{(0)}(i)}{x^{(0)}(i)}\right|\right]^{\frac{1}{N}} \times 100
\end{gathered}
$$

where $\hat{x}^{(0)}(i)$ is the forecasting result obtained from the benchmark method. In this case, it is the worst forecasting result to $x^{(0)}(i)$ of all the three models.

Despite having similar functions of determining which is the best model, these error analysis indicators have fine distinctions. The MAPE is an accuracy indicator that reflects the general closeness of the forecasting results to the RD. The MdAPE is the middle value of all absolute percentage errors ordered by size and can also reflect the general closeness of the forecasting results to the RD. In addition, the MdAPE can overcome the influence of several outliers. The MaxAPE generates the worst forecasting result and reflects the maximal forecasting risk. Compared with the other three indicators, the GMARE pays more attention to relative superiority.

Using data in Table 1, Eq. (12), and Eqs. (17) to (19), each error analysis indicator for each forecasting model was calculated (see Table 2).

As shown in Table2, ONEM is always much better than the other two forecasting models for each error analysis indicator. This result proves the applicability of ONEM in forecasting the yearly electricity consumption of developing countries like India.

\section{Conclusions}

Yearly electricity consumption forecasting is very important for electricity price adjustment and system expansion planning. For most developing countries, yearly electricity consumption trends are approximately exponential growth curves. The ONEM is proposed in this paper as a method of forecasting the yearly electricity consumption by using trend extrapolation. The nonhomogeneous exponential forecasting equation is obtained by using the IAGO, discretizing the differential equation, minimizing the RSS, and accumulating the intermediate homogeneous exponential equation. Furthermore, the PSO algorithm is used to optimize the equation parameters. To evaluate the forecasting performance of the model, the $\operatorname{ONEM}, \operatorname{GM}(1,1)$, and RBFANN are all used to forecast India's yearly electricity consumption from 2001 to 2010. Results show that the MAPE, MdAPE, MaxAPE, and GMARE of ONEM are as small as $3.84 \%, 2.92 \%, 8.13 \%$, and $23.31 \%$ respectively, which are much better than the results obtained by the other two models.

\section{Acknowledgement}

This study was supported by the National Natural Science Foundation of China (NSFC) (71201057 and 71071052) and the Fundamental Research Funds for the Central Universities (11MR57).

The authors are grateful to the anonymous referee for a careful checking of the details and for helpful comments that improved this paper.

\section{References}

[1] A. E. Akinlo, "Electricity consumption and economic growth in nigeria: evidence from cointegration and co-feature analysis," Journal of Policy Modeling, 31, 681-693 (2009).

[2] S.-H. Yoo and S.-Y. Kwak, "Electricity consumption and economic growth in seven south american countries," Energy Policy, 38, 181-188 (2010).

[3] M. Shahbaz and H. H. Lean, "The dynamics of electricity consumption and economic growth: A revisit study of their causality in pakistan," Energy, 39, 146-153 (2012). 
[4] H. Gurgul and $€$. Lach, "The electricity consumption versus economic growth of the polish economy," Energy Economics, 34, 500-510 (2012).

[5] L. J. Soares and M. C. Medeiros, "Modeling and forecasting short-term electricity load: A comparison of methods with an application to brazilian data," International Journal of Forecasting, 24, 630-644 (2008).

[6] H. Kebriaei, B. N. Araabi, and A. Rahimi-Kian, "Shortterm load forecasting with a new nonsymmetric penalty function," Power Systems, IEEE Transactions on, 26, 18171825 (2011).

[7] D.-x. Niu, H.-f. Shi, and D. D. Wu, "Short-term load forecasting using bayesian neural networks learned by hybrid monte carlo algorithm," Applied Soft Computing, 12, 18221827 (2012).

[8] C. Guan, P. B. Luh, L. D. Michel, Y. Wang, and P. B. Friedland, "Very short-term load forecasting: Wavelet neural networks with data pre-filtering, (2013).

[9] O. A. Carpinteiro, R. C. Leme, A. C. Z. de Souza, C. A. Pinheiro, and E. M. Moreira, "Long-term load forecasting via a hierarchical neural model with time integrators," Electric Power Systems Research, 77, 371-378 (2007).

[10] S. Ben Taieb, G. Bontempi, A. F. Atiya, and A. Sorjamaa, "A review and comparison of strategies for multi-step ahead time series forecasting based on the nn5 forecasting competition," Expert systems with applications, 39, 7067-7083 (2012).

[11] L. Ghelardoni, A. Ghio, and D. Anguita, "Energy load forecasting using empirical mode decomposition and support vector regression, (2013).

[12] M. Meng and D. Niu, "Annual electricity consumption analysis and forecasting of china based on few observations methods," Energy Conversion and Management, 52, 953-957 (2011).

[13] J. Moreira Pessanha and N. Leon, "Long-term forecasting of household and residential electric customers in brazil," Latin America Transactions, IEEE (Revista IEEE America Latina), 10, 1537-1543 (2012).

[14] Á. L. Santana, G. B. Conde, L. P. Rego, C. A. Rocha, D. L. Cardoso, J. C. Costa, U. H. Bezerra, and C. R. Francês, "Predict-decision support system for load forecasting and inference: A new undertaking for brazilian power suppliers," International Journal of Electrical Power \& Energy Systems, 38, 33-45 (2012).

[15] P. Zhou, B. Ang, and K. Poh, "A trigonometric grey prediction approach to forecasting electricity demand," Energy, 31, 2839-2847 (2006).

[16] A. Azadeh, S. Ghaderi, and S. Sohrabkhani, "Annual electricity consumption forecasting by neural network in high energy consuming industrial sectors," Energy Conversion and Management, 49, 2272-2278 (2008).

[17] Y. Wang, J. Wang, G. Zhao, and Y. Dong, "Application of residual modification approach in seasonal arima for electricity demand forecasting: A case study of china," Energy Policy, 48, 284-294 (2012).

[18] B. Petroleum, "Bp statistical review of world energy," Retrieved September, 9, 2008 (2008).

[19] O. A. Carpinteiro, A. J. Reis, and A. da Silva, "A hierarchical neural model in short-term load forecasting," Applied Soft Computing, 4, 405-412 (2004).

[20] H. S. Hippert and J. W. Taylor, "An evaluation of bayesian techniques for controlling model complexity and selecting inputs in a neural network for short-term load forecasting," Neural networks, 23, 386-395 (2010).

[21] E. González-Romera, M. Jaramillo-Morán, and D. Carmona-Fernández, "Monthly electric energy demand forecasting with neural networks and fourier series," Energy Conversion and Management, 49, 3135-3142 (2008).

[22] M. Meng, D. Niu, and W. Sun, "Forecasting monthly electric energy consumption using feature extraction," Energies, 4, 1495-1507 (2011)

[23] D. Ju-Long, "Control problems of grey systems," Systems \& Control Letters, 1, 288-294 (1982).

[24] M. Meng, D. Niu, and W. Shang, "Coi subi $2 ; / \operatorname{sub}_{i}$ emissions and economic development: China's 12th five-year plan," Energy Policy, 42, 468-475 (2012).

[25] W. Zhou and J.-M. He, "Generalized gm $(1,1)$ model and its application in forecasting of fuel production," Applied Mathematical Modelling, (2013).

[26] J. Kennedy, "Particle swarm optimization," in Encyclopedia of Machine Learning. Springer, 760-766 (2010).

[27] M. Sadeghpour, H. Salarieh, G. Vossoughi, and A. Alasty, "Multi-variable control of chaos using pso-based minimum entropy control," Communications in Nonlinear Science and Numerical Simulation, 16, 2397-2404 (2011).

[28] A. Yadav and K. Deep, "Shrinking hypersphere based trajectory of particles in pso," Applied Mathematics and Computation, 220, 246-267 (2013).

[29] F. Abbas and N. Choudhury, "Electricity consumptioneconomic growth nexus: An aggregated and disaggregated causality analysis in india and pakistan," Journal of Policy Modeling, (2012).

[30] S. R. Rallapalli and S. Ghosh, "Forecasting monthly peak demand of electricity in indiała critique," Energy Policy, 45, 516-520 (2012).

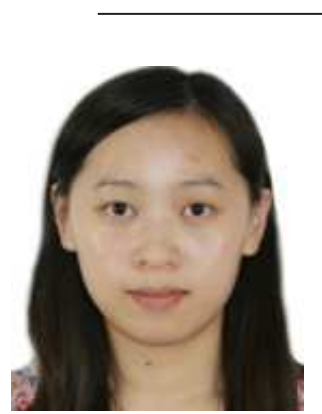

Xiaomin $\mathbf{X u}$ is a $\mathrm{PhD}$ student of the School of Economics and Management, North China Electric Power University, Beijing, China. Her thesis is about power load forecasting and project management.

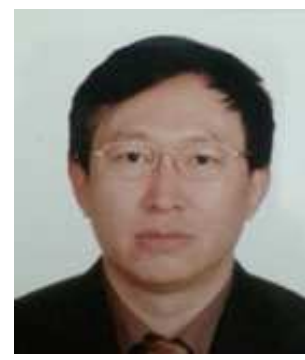

Dongxiao Niu, $\mathrm{PhD}$, is a Professor of North China Electric Power University, Beijing, China. His interesting research areas are power load forecasting and comprehensive evaluation. He has published several scientific papers in reputed international journals. 


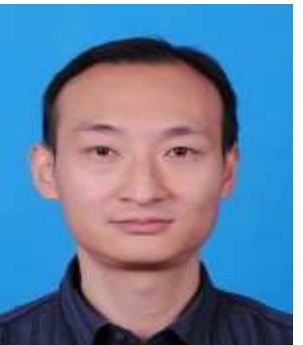

Ming Meng, $\mathrm{PhD}$, is a teacher of North China Electric Power University, Baoding, China. His research interests are in the areas of model and policy of energy composition and $\mathrm{CO} 2$ emissions. He has published several research articles in reputed international journals of these areas. He is a referee of 8 international journals.

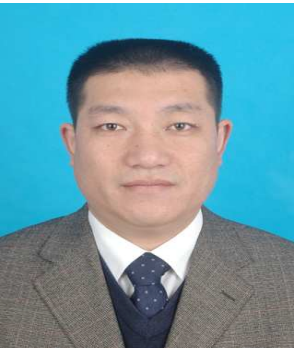

Huifeng Shi is Associate Professor of Mathematical at North China Electric Power University Baoding, China. $\mathrm{He}$ received the $\mathrm{PhD}$ degree in "Management" at North China Electric Power University . His research interests are in the areas of forecasting approaches and

Bayesian statistics methods. 